\title{
Automatic Detection of Air Holes inside the Esophagus in CT Images
}

\author{
Andreas Fieselmann ${ }^{1,2}$, Stefan Lautenschläger ${ }^{2}$, Frank Deinzer ${ }^{2}$, Björn Poppe $^{1}$ \\ ${ }^{1}$ Medical Radiation Physics, University of Oldenburg, Oldenburg, Germany \\ ${ }^{2}$ Siemens AG Medical Solutions, Forchheim, Germany \\ andreas.fieselmann.ext@siemens.com
}

\begin{abstract}
Air holes inside the esophagus can be used to localize the esophagus in computed tomographic (CT) images. In this work we present a technique to automatically detect esophageal air holes in this modality. Our technique is based on the extraction of a volume of interest, air segmentation by thresholding and classification of respiratory and esophageal air using a priori knowledge about the connectivity of air voxels. A post-processing step rejects wrong results from artifacts in the CT image. We successfully tested our algorithm with clinical data and compared the detection results of a human expert and our technique.
\end{abstract}

\section{Introduction}

The esophagus is a muscular tube with a diameter of approximately $10 \mathrm{~mm}$. It passes from the pharynx through the mediastinum into the stomach and has direct contact with the left atrial posterior wall whereas the exact route varies individually [1]. It is important to know the position of the esophagus during atrial ablation therapy. This intervention treats atrial fibrillation by radiofrequency catheter ablation of the pulmonary veins inside the left atrium. If the power of the catheter at the contact area of the left atrium and the esophagus is too high, thermal injury of the esophagus may occur and a fistula that connects the esophagus and the left atrium can develop [2]. This situation is potentially fatal because air is able enter into the left atrium.

A segmentation of the esophagus is helpful to plan the ablation strategy and decrease the power at its contact area with the left atrium. Unfortunately, the contrast of the esophagus in medical images (cardiac CT, cardiac C-arm CT [3] or cardiac MRI) is low which makes edge-based segmentation difficult. A feature to identify the position of the esophagus are small air holes inside the esophagus (Fig. 1). They are a normal phenomenon and are visible in most CT images, although their number and sizes are highly variable.

Very few studies investigated the segmentation of the esophagus in tomographic images $[4,5,6]$. Rousson et al. [4] detect esophageal air holes to refine their segmentation result. After their segmentation algorithm has estimated a centerline of the esophagus low intensity voxels are detected in the neighborhood of the centerline. The complete air holes are obtained by means of a region growing algorithm. Two points on the centerline and a segmentation of the aorta and 
left atrium are needed as input to the algorithm. The two other studies $[5,6]$ use optic flow and deformable models respectively to segment the esophagus but do not detect esophageal air holes. Our technique detects esophageal air holes fully automatic without any user input and can be used to initialize an algorithm to segment the esophagus.

\section{Materials and Methods}

Our algorithm consists of three main steps which are described in the following sections and illustrated in Fig. 2.

\subsection{Automated Extraction of a Volume of Interest}

The algorithm confines all computations to a volume of interest (VOI) in order to decrease computational time and the number of wrong results in the subsequent analysis. It is extracted relative to a reference point $\boldsymbol{p}=\left(p_{x}, p_{y}, p_{z}\right)^{T}$ at the left atrium which is computed by projecting the center of mass of the left atrium onto its posterior wall. A segmentation of the left atrium is available because it is the primary organ of interest and always segmented before the esophagus in the clinical workflow.

We use a $2 \mathrm{D}$ rectangular region of interest centered at $\left(p_{x}+t_{x}, p_{y}+t_{y}\right)^{T}$ in all axial slices. The side lengths $s_{x}, s_{y}$ and $\boldsymbol{t}=\left(t_{x}, t_{y}\right)^{T}$ are determined from a set of CT images such that the esophagus is always inside the VOI. Our parameters measured in $\mathrm{mm}$ and with orientation as shown in Fig. 1 are $\boldsymbol{t}=(-15,-20)^{T}$, $s_{x}=100$ and $s_{y}=100$.

\subsection{Classification of Esophageal Air Holes}

Most visible air in a cardiac CT scan exists inside the respiratory organs: the lungs, the bronchi and the trachea. This respiratory air constitutes one connected

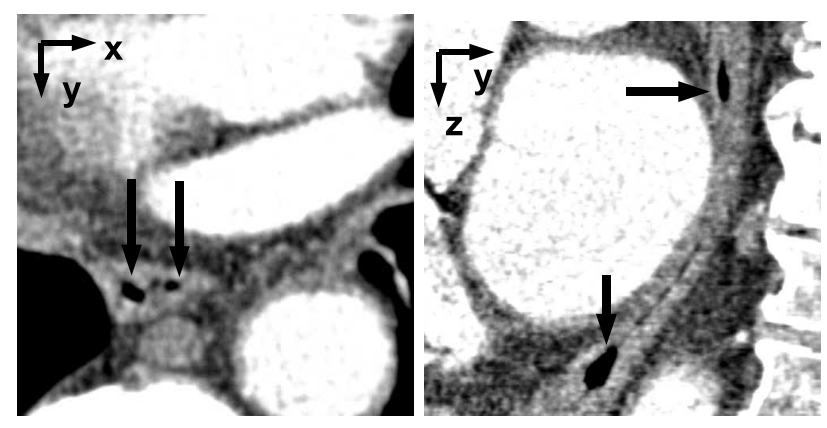

(a)

(b)

Fig. 1. Cardiac CT images with esophageal air holes: (a) axial and (b) sagittal view 
component. Another location in the mediastinum where air can exist is inside the esophagus.

Air has a very low Hounsfield unit (HU) value in CT images. In our algorithm the entire air is segmented by applying a threshold value $I_{\text {air }}$ at $-400 \mathrm{HU}$. This threshold value has been suggested by Kemerink et al. [7] to segment the lung parenchyma. All voxels with intensities below $-400 \mathrm{HU}$ are labeled as air.

In the next step the air is classified into respiratory or esophageal air. The respiratory air is classified using a 3D volume growing algorithm [8] whose seeds are placed at the left and right boundaries of the VOI at voxels that have been labeled as air. The output of the volume growing algorithm is subtracted from the initial air image. In the resulting image all air voxels not connected to the boundaries are still labeled. These voxels are classified as esophageal air. Finally, esophageal air holes are found by identifying connected components of esophageal air.

\subsection{Post-processing}

Artifacts in the CT image with low HU values can be misclassified as esophageal air. An important example are dark streaks due to beam hardening of the X-rays [9]. They occur very close to high intensity areas. These high intensity areas originate e.g. from the use of a contrast agent. Post-processing is needed to recognize and reject artifacts misclassified as esophageal air.

Our algorithm recognizes beam hardening artifacts by computing the image intensity histogram in a certain region around each esophageal air hole. If the histogram contains intensities greater than $600 \mathrm{HU}$ then the air hole is rejected. The histogram region is determined by dilating the bounding box of the air hole isotropically by $5 \mathrm{~mm}$.

\section{Results}

We validate our algorithm with 7 pre-interventional contrast-enhanced cardiac CT scans of patients treated with atrial ablation therapy. The scans were ac-

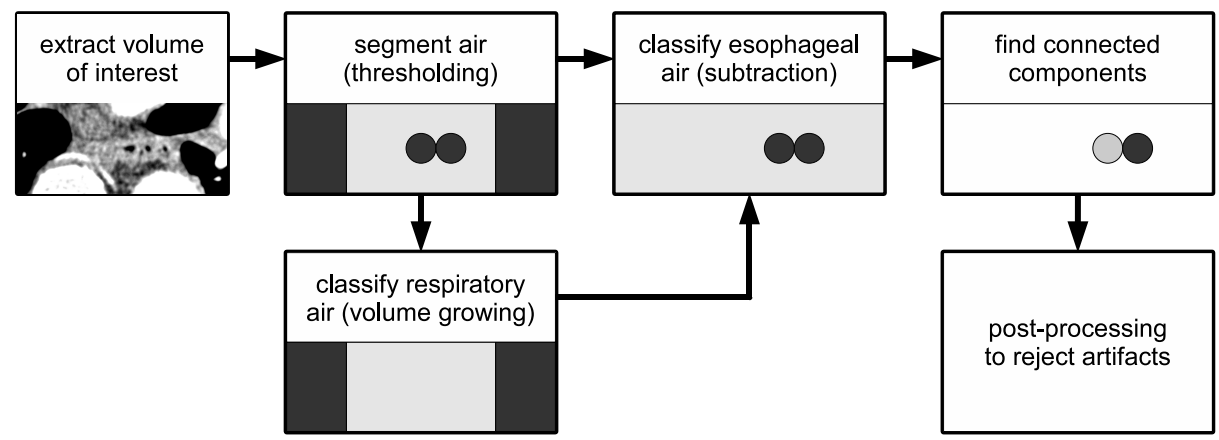

Fig. 2. Flow chart of the algorithm to detect esophageal air holes in a CT image 
Table 1. Esophageal air holes detected by a human expert and the algorithm in 7 patient scans. Caudal and cranial are defined relative to the left atrial center

\begin{tabular}{llllllll}
\hline patient number & 1 & 2 & 3 & 4 & 5 & 6 & 7 \\
\hline $\begin{array}{l}\text { human expert } \\
\text { number at caudal/cranial side }\end{array}$ & $3 / 9$ & $11 / 11$ & $5 / 2$ & $10 / 4$ & $6 / 3$ & $4 / 5$ & $2 / 1$ \\
$\begin{array}{l}\text { algorithm } \\
\text { number at caudal/cranial side }\end{array}$ & $3 / 2$ & $4 / 4$ & $5 / 1$ & $7 / 0$ & $4 / 0$ & $0 / 1$ & $2 / 0$ \\
misclassified esoph. air holes & 0 & 0 & 1 & 0 & 0 & 0 & 0 \\
number of rejected artifacts & 19 & 16 & 0 & 25 & 8 & 41 & 4 \\
minimum size in voxels & 1 & 1 & 3 & 1 & 1 & 8 & 1 \\
maximum size in voxels & 6623 & 1859 & 9344 & 65 & 1694 & 8 & 3506 \\
\hline
\end{tabular}

quired using a 16-slice MDCT scanner (SOMATOM ${ }^{\circledR}$ Sensation 16, Siemens AG Medical Solutions) with an in-plane resolution of approximately $0.35 \cdot 0.35 \mathrm{~mm}^{2}$ and a slice thickness of approximately $0.6 \mathrm{~mm}$. A human expert labels all esophageal air holes manually and the left atrium is segmented using the software syngo ${ }^{\circledR}$ InSpace EP (Siemens AG Medical Solutions). We implement our algorithm in $\mathrm{C}++$ using the open-source software library Insight Toolkit [10].

Our algorithm detects esophageal air holes in all 7 patient scans (Table 1) but misses air holes that have intensities greater than $I_{\text {air }}$ which are found by the human expert. The number of misclassified air holes is very low and all beam hardening artifacts are successfully recognized and rejected. The sizes of detected air holes vary strongly. The minimum size is one pixel volume but this does not correspond to the true size of the air hole which contains some voxel intensities greater than $I_{\text {air }}$. The majority of air holes are found caudal to the left atrial center before the esophagus enters into the stomach. Fig. 3 shows air holes cranial (a-c) and caudal (d-f) to the left atrial center. The computational time is about 5 seconds for one patient scan.

\section{Discussion}

Our algorithm successfully detects esophageal air holes in cardiac CT scans and requires no input from the user. It makes use of the connectivity of respiratory air voxels which are not connected to esophageal air voxels. A possible extension of our algorithm is to use an adaptive threshold to segment the air. Because only a few air holes are sufficient to localize the esophagus it is not a high priority to detect all air holes.

The locations of air holes reveal the position of the esophageal lumen and the contour of the esophagus can be estimated using this knowledge. It is possible to visualize the air holes directly or use the air holes to initialize an algorithm to segment the esophagus. 
Fig. 3. Regions of cardiac CT images with labeled esophageal air holes: (a-c) cranial of the atrial center, (d-f) caudal of the atrial center

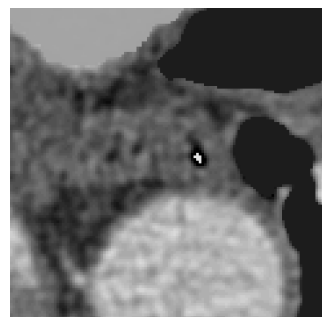

(a)

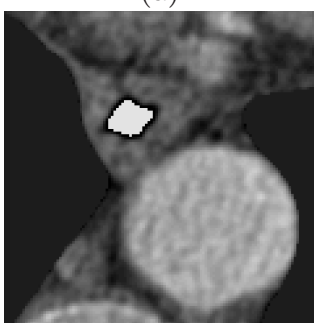

(d)

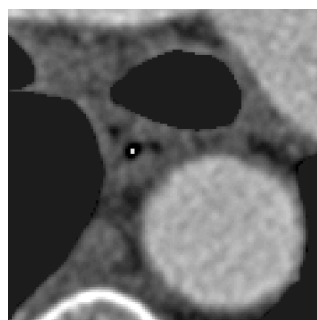

(b)

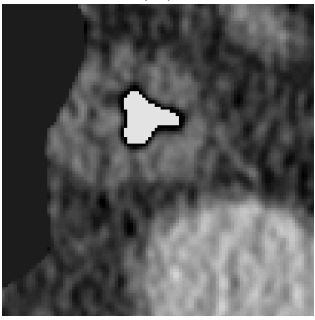

(e)

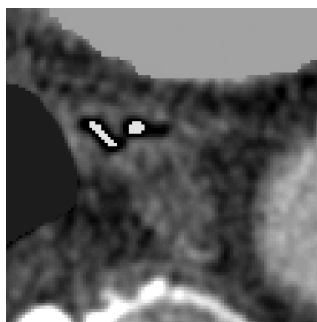

(c)

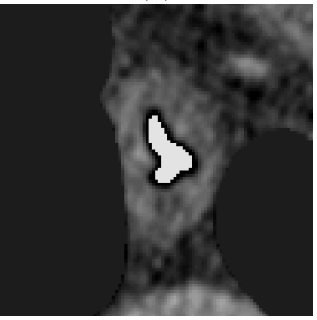

(f)

\section{References}

1. Tsao HM, Wu MH, Higa S, et al. Anatomic relationship of the esophagus and left atrium: Implication for catheter ablation of atrial fibrillation. Chest. $2005 ; 128(4): 2581-7$.

2. Pappone C, Oral H, Santinelli V, et al. Atrio-esophageal fistula as a complication of percutaneous transcatheter ablation of atrial fibrillation. Circulation. 2004;109(22):2724-6.

3. Lauritsch G, Boese J, Wigström L, et al. Towards cardiac C-arm computed tomography. IEEE Trans Med Imaging. 2006;25(7):922-34.

4. Rousson M, Bai Y, Xu C, et al. Probabilistic minimal path for automated esophagus segmentation. Proc SPIE. 2006;6144:49-4H.

5. Huang TC, Zhang G, Guerrero T, et al. Semi-automated CT segmentation using optic flow and Fourier interpolation techniques. Comput Methods Programs Biomed. 2006;84(2-3):124-34.

6. Ragan D, Starkschall G, McNutt T, et al. Semiautomated four-dimensional computed tomography segmentation using deformable models. Med Phys. $2005 ; 32(7): 2254-61$.

7. Kemerink GJ, Lamers RJS, Pellis BJ, et al. On segmentation of lung parenchyma in quantitative computed tomography of the lung. Med Phys. 1998;25(12):2432-9.

8. Handels H. Medizinische Bildverarbeitung. Stuttgart: Teubner; 2000.

9. Kalender WA. Computed Tomography. 2nd ed. Erlangen: Publicis Corporate Publishing; 2005.

10. Ibanez L, Schroeder W, Ng L, et al. The ITK Software Guide. 2nd ed. Kitware Inc.; 2005. 